\title{
HIGH DYNAMIC PRECISION CONTROL SYSTEM
}

\section{Y. A. VERSHININ}

Coventry University, Coventry, England

\begin{abstract}
This paper is dealing with the design of a high precision adaptive control system for multivariable systems or processes. It is the well -known fact that the desired behavior of local sub-systems in a multivariable system is difficult to obtain due to the interconnections between channels. The method, presented in this paper, allows one to decouple channels and to adjust the desirable performance of the overall system according to reference models of local sub-systems. The stability of the overall system is guaranteed following the stability criteria based on the Lyapunov function
\end{abstract}

KEYWORDS: Control Engineering, Dynamic Systems, Adaptive Control \& Stability of Systems

Received: Apr 24, 2018; Accepted: May 14, 2018; Published: Jun 01, 2018; Paper Id.: IJEEERJUN20182

\section{INTRODUCTION}

Many industrial processes and systems include several inputs and several outputs. A mathematical model of such a processor system can be represented in the form of a MIMO system (Many-Inputs-Many-Outputs) [1] and [2]. For example a chemical batch reactor with several inputs and outputs. Another example is an airplane, where the pitch, roll and yaw angles can be controlled by a pilot. Yet another example is a robot manipulator where the motion of each joint can be controlled independently. Due to the interconnection between channels via the system's dynamics, the response of the system may not follow for the desiredtrajectory of the applied input. In order to solve this problem, decoupled control algorithms are suggested to use. However, parameters of real systems can change due to the change of environmental conditions, for example, due to the change of the temperature of the environment. Therefore, the outputs of real systems will also change. It is suggested in this paper to employ adaptive control algorithms in order to provide the system's behavior, according to the reference model for each channel. It is admitted that the dynamics of different channels can also be different. Therefore, the relevant reference models for each channel can be employed in the multivariable system. The stability of the overall multivariable system is guaranteed according to the obtained adaptive control algorithms based on the Lyapunov stability criterion.

\section{THE TASK FORMULATION}

A multivariable plant is described by the system of matrix differential equations [1]:

$$
\begin{aligned}
& \dot{X}_{1}=A_{11} X_{1}+A_{12} X_{2}+\ldots+A_{1 n} X_{n}+U_{1}, \\
& \dot{X}_{2}=A_{21} X_{1}+A_{22} X_{2}+\ldots+A_{2 n} X_{n}+U_{2}, \\
& \dot{X}_{n}=A_{n 1} X_{1}+A_{n 2} X_{2}+\ldots+A_{n n} X_{n}+U_{n},
\end{aligned}
$$


where

$X_{1}=\left[x_{11}, x_{12}, \ldots, x_{1 m}\right]^{T}$,

$X_{2}=\left[x_{21}, x_{22}, \ldots, x_{2 v}\right]^{T}$,

$X_{n}=\left[x_{n 1}, x_{n 2}, \ldots, x_{n k}\right]^{T}$.

$X_{1}, X_{2}, \ldots, X_{n}$-are vectors of states of the plant,

$A_{11}=A_{11}(t), A_{12}=A_{12}(t), \ldots, A_{n n}=A_{n n}(t)$-are matrices of variable paramerers of the plant,

$U_{1}, U_{2}, \ldots, U_{n}-$ are control vectors.

It is required to find such feedback laws that autonomous motion of plant's coordinates at each channel will be provided according to the following reference model:

$\dot{X}_{1}^{m}=A_{11}^{m} X_{1}^{m}+G_{1}$,

$\dot{X}_{2}^{m}=A_{22}^{m} X_{2}^{m}+G_{2}$,

$\dot{X}_{n}^{m}=A_{n n}^{m} X_{n}^{m}+G_{n,}$

where

$X_{1}^{m}=\left[x_{11}^{m}, x_{12}^{m}, \ldots, x_{1 m}^{m}\right]^{T}$,

$X_{2}^{m}=\left[x_{21}^{m}, x_{22}^{m}, \ldots, x_{2 v}^{m}\right]^{T}$,

$X_{n}^{m}=\left[x_{n 1}^{m}, x_{n 2}^{m}, \ldots, x_{n k}^{m}\right]^{T}$.

$X_{1}^{m}, X_{2}^{m}, \ldots, X_{n}^{m}$-are vectors of states of the reference $\bmod e l$,

$A_{11}^{m}, A_{22}^{m}, \ldots, A_{n n}^{m}$-are matrices of paramerers of the reference $\bmod e l$,

$G_{1}, G_{2}, \ldots, G_{n}-$ are inputs.

\section{Synthesis of the Basic Loop's Structure}

The feedback controls can be chosen as follows:

$U_{1}=G_{1}+K_{11} X_{1}+K_{12} X_{2}+\ldots+K_{1 n} X_{n}$

$U_{2}=G_{2}+K_{21} X_{1}+K_{22} X_{2}+\ldots+K_{2 n} X_{n}$,

$U_{n}=G_{n}+K_{n 1} X_{1}+K_{n 2} X_{2}+\ldots+K_{n n} X_{n}$

where

$K_{11}=K_{11}(t), K_{12}=K_{12}(t), \ldots, K_{n n}=K_{n n}(t)$-are matrices of the feedback variable gains.

According to the equations (1) and (3), the closed-loop system can be represented as follows: 


$$
\begin{aligned}
& \dot{X}_{1}=\left(A_{11}+K_{11}\right) X_{1}+\left(A_{12}+K_{12}\right) X_{2}+\ldots+\left(A_{1 n}+K_{1 n}\right) X_{n}+G_{1}, \\
& \dot{X}_{2}=\left(A_{21}+K_{21}\right) X_{1}+\left(A_{22}+K_{22}\right) X_{2}+\ldots+\left(A_{2 n}+K_{2 n}\right) X_{n}+G_{2},
\end{aligned}
$$

$$
\dot{X}_{n}=\left(A_{n 1}+K_{n 1}\right) X_{1}+\left(A_{n 2}+K_{n 2}\right) X_{2}+\ldots+\left(A_{n n}+K_{n n}\right) X_{n}+G_{n},
$$

It can be seen from (4) that the system "plant + controller" will be dynamically decoupled on each channel if the following conditions are achieved:

$$
A_{i j}+K_{i j}=0, \quad(\mathrm{i}, \mathrm{j}=1, \ldots \mathrm{n}, \quad \mathrm{i} \neq \mathrm{j})
$$

In order to guarantee the desired motion of the system according to the reference trajectories (2), it needs to provide the following relation:

$$
A_{i i}+K_{i i}=A_{i i}^{m}
$$

Thus, for the accomplishment of (5) and (6) the following should be provided:

$$
\begin{aligned}
& a_{12,0}+k_{12,0}=0, \\
& a_{12,1}+k_{12,1}=0, \\
& a_{12, m-1}+k_{12, m-1}=0, \\
& a_{n n-1, k-1}+k_{n n-1, k-1}=0, \\
& \left(a_{11,0}+k_{11,0}\right)=a_{11,0}^{m}, \\
& \left(a_{11,1}+k_{11,1}\right)=a_{11,1}^{m}, \\
& \left(a_{11, m-1}+k_{11, m-1}\right)=a_{11, m-1}^{m}, \\
& \left(a_{n n, m-1}+k_{m, k-1}\right)=a_{m, k-1}^{m},
\end{aligned}
$$

where

$a_{11,0}=a_{11,0}(t), a_{11,1}=a_{11,1}(t), \ldots, a_{n n, k-1}=a_{n n, k-1}(t)-$ are var iable scalar coefficients of the plant (1),

$a_{11,0}^{m}, a_{11,1}^{m}, \ldots, a_{n n, k-1}^{m}$-are cons $\tan t$ scalar coefficients of the $\bmod e l(2)$,

$k_{11,0}=k_{11,0}(t), k_{11,1}=k_{11,1}(t), \ldots, k_{n n, k-1}=k_{n n, k-1}(t)$-are variable scalar gains of the controller (3).

\section{Synthesis of the Adaptive Control Algorithms}

The variable parameters of the plant (1) can be represented as follows:

$$
\begin{aligned}
& A_{11}=A_{11}^{0}+\Delta A_{11}, \\
& A_{12}=A_{12}^{0}+\Delta A_{12}, \\
& A_{n n}=A_{n n}^{0}+\Delta A_{n n},
\end{aligned}
$$

\section{Where}


$A_{i j}^{0}=$ const $, \quad(\mathrm{i}, \mathrm{j}=1, \ldots \mathrm{n}), \quad A_{i j}^{0}$-are the cons $\tan t$ matrices whose coefficients correspond to the no minal operation mode of the plant,

$\Delta A_{i j}=\Delta A_{i j}(t), \quad \Delta A_{i j}$-are deviation of the plant parameters from their no min al operation mode.

The controller parameters can be represented analogously:

$K_{11}=K_{11}^{0}+\Delta K_{11}$,

$K_{12}=K_{12}^{0}+\Delta K_{12}$,

$K_{n n}=K_{n n}^{0}+\Delta K_{n n}$

where

$K_{i j}^{0}=$ const $, \quad(\mathrm{i}, \mathrm{j}=1, \ldots \mathrm{n}), \quad K_{i j}^{0}$-are matrices of cons $\tan t$ coefficients of the controller,

$\Delta K_{i j}=\Delta K_{i j}(t), \quad \Delta K_{i j}$-are matrices of adjusting coefficients of the controller.

Thus, the motion of plant coordinates on a non-nominal operation mode can be represented as follows:

$\dot{X}_{i}=\sum_{j=1}^{n}\left(A_{i j}^{0}+\Delta A_{i j}(t)\right) X_{j}+U_{i,} \quad(i=1, \ldots, n)$.

The feedback control is:

$U_{i}=G_{i}+\sum_{j=1}^{n}\left(K_{i j}^{0}+\Delta K_{i j}(t)\right) X_{j}, \quad(i=1, \ldots, n)$.

Substituting the equation (9) into (8) we can obtain the equation of motion of the system "the plant + the controller" on each channel:

$\dot{X}_{i}=\sum_{j=1}^{n}\left(A_{i j}^{0}+\Delta A_{i j}(t)\right) X_{j}+\sum_{j=1}^{n}\left(K_{i j}^{0}+\Delta K_{i j}(t)\right) X_{j}+G_{i}$

The model motion equation on each channel can be chosen as follows:

$$
\dot{X}_{i}^{m}=A_{i i}^{m} X_{i}^{m}+\sum_{\substack{j=1 \\ j \neq i}}^{n}\left(A_{i j}^{0}+K_{i j}^{0}\right) X_{j}+G_{i}
$$

Comparing (10) and (11) and taking into account that $A_{i i}^{0}+K_{i i}^{)}=A_{i i}^{m}$, we can obtain:

$$
\dot{E}_{i}=A_{i i}^{m} E_{i}+\left(\Delta A_{i i}(t)+\Delta K_{i i}(t)\right) X_{i}+\left(\sum_{\substack{j=1 \\ j \neq i}}^{n} \Delta A_{i j}(t)+\sum_{\substack{j=1 \\ j \neq i}}^{n} \Delta K_{i j}(t)\right) X_{j}
$$

where

$E_{i}=X_{i}-X_{i}^{m}$ 
Let us introduce the following notation:

$Y_{i i}=Y_{i i}(t)=\Delta A_{i i}(t)+\Delta K_{i i}(t)$,

$Y_{i j}=Y_{i j}(t)=\Delta A_{i j}(t)+\Delta K_{i j}(t), \quad(i \neq j)$.

Thus, the error equation (12) can be represented as:

$\dot{E}_{i}=A_{i i}^{m} E_{i}+Y_{i i} X_{i}+\sum_{\substack{j=1 \\ j \neq i}}^{n} Y_{i j} X_{j}$

Algorithms of adaptation can be specified in the following form:

$\frac{d}{d t} \Delta K=\Psi$

Admit, that parameters' deviation $\Delta A=\Delta A(t)$ is differentiable in time:

$\frac{d}{d t} \Delta A(t)=R(t)$

Therefore, according to (13) - (17) we obtain:

$\dot{E}=A^{m} E+Y X$,

$\dot{Y}=\Psi+R$.

where $R=R(t)$

The equations (18) with eq. (2) Describe the dynamic motion of the adaptive control system with the reference model.

Admit, that the matrix $\Psi$ is a function of error deviation $E$ and time $t$, and $\Psi(E, t)=0$ at $t=0$.

In this case the state and parametric error motion

$E \equiv 0, \quad Y \equiv 0$

With the admission that $R(t) \equiv 0$ according to the hypothesis of quasi-stability, is the solution of the system (18).

It is suggested to use the second method of Lyapunov in order to obtain the adaptation algorithms $\Psi$ from the condition of stability of the zero solution (19) of the system (18)) [3], [4], [5] and [6].

The quadratic function $\mathrm{V}$ can be chosen as follows:

$V=\gamma E^{T} P E+\operatorname{tr}\left(Y Y^{T}\right)$,

where

$\gamma=$ const

$P-i$ simmetric matrix. 
$Q-i s$ negative definite matrix

A time derivative for the function $\mathrm{V}$ is obtained as follows:

$$
\begin{aligned}
& \dot{V}=\gamma \dot{E}^{T} P E+\gamma E^{T} P \dot{E}+\operatorname{tr}\left(\dot{Y} Y^{T}+Y \dot{Y}^{T}\right)=\gamma\left(A^{m} E+Y X\right)^{T} P E+\gamma E^{T} P\left(A^{m} E+Y X\right)+\operatorname{tr}\left(\dot{Y} Y^{T}+Y \dot{Y}^{T}\right)= \\
& \gamma\left[\left(A^{m} E\right)^{T}+(Y X)^{T}\right] P E+\gamma E^{T} P\left(A^{m} E+Y X\right)+\operatorname{tr}\left(\dot{Y} Y^{T}+Y \dot{Y}^{T}\right)= \\
& \gamma\left[E^{T}\left(A^{m}\right)^{T}+X^{T} Y^{T}\right] P E+\gamma E^{T} P A^{m} E+\gamma E^{T} P Y X+\operatorname{tr}\left(\dot{Y} Y^{T}+Y \dot{Y}^{T}\right)= \\
& \gamma E^{T}\left(A^{m}\right)^{T} P E+\gamma X^{T} Y^{T} P E+\gamma E^{T} P A^{m} E+\gamma E^{T} P Y X+\operatorname{tr}\left(\dot{Y} Y^{T}+Y \dot{Y}^{T}\right)= \\
& \gamma E^{T}\left(\left(A^{m}\right)^{T} P+P A^{m}\right) E+\gamma X^{T} Y^{T} P E+\gamma E^{T} P Y X+\operatorname{tr}\left(\dot{Y} Y^{T}+Y \dot{Y}^{T}\right)
\end{aligned}
$$

After some intermediate matrix manipulations we obtain that:

$$
\Psi=-\gamma P E X^{T}
$$

The derivative of the Lyapunov function is represented as:

$\dot{V}=\gamma E^{T} Q E$

Taking into account that $A^{T}$ is Hurvitch matrix, we obtain:

$$
\dot{V} \leq 0
$$

Therefore, according to (18), (16) and using conditions of quasi-stationarity of the system, it is straightforward to obtain the adaptation algorithms as:

$$
\Delta \dot{K}=-\gamma P E X^{T}
$$

It follows from the above that the motion of the system (16) with the algorithms (25) is stable and conditions (5) and (6) are satisfied.

\section{CONCLUSIONS}

The developed in this paper control algorithms allow one to improve the performance of a multivariable process or system due to the decoupling of channels of the process or system from the interaction via the process's or system's dynamics. The desirable performance of the process or system can be obtained using the relevant reference models for each channel. Adaptive control algorithms allow one to adjust the overall system's response in the case of parameters change. The stability of the overall system is guaranteed according to the Lyapunov stability criterion.

\section{REFERENCES}

1. P.K. Sinha, Multivariable Control: An Introduction, Publisher: Marcel Dekker: NY, 1984.

2. Norman S. Nise, Control Systems Engineering, Wiley, 2015.

3. Shankar Sastry and Marc Bodson, Adaptive Control: Stability, Convergence, and Robustness, Dover Publications, 2011.

4. P.V. Kokotovic (Ed.), Foundation of Adaptive Control, Springer-Verlag, 1991.

5. B. Egardt, Stability of Adaptive Controllers, Springer 2014.

6. Keigo Watanabe, Adaptive Estimation and Control: Partitioning Approach. Prentice Hall International, 1992. 